\title{
Implications of Plasmodium glycosylation on vaccine efficacy $\&$ design
}

\author{
Ethan D Goddard-Borger*,1,2 \& Justin A Boddey**,1,2 \\ ${ }^{1}$ The Walter \& Eliza Hall Institute of Medical Research, 1G Royal Parade, Parkville, Victoria 3052, Australia \\ ${ }^{2}$ Department of Medical Biology, University of Melbourne, Parkville, Victoria 3010, Australia \\ *Author for correspondence: goddard-borger.e@wehi.edu.au \\ **Author for correspondence: boddey@wehi.edu.au
}

\begin{abstract}
"Beyond the small O- and C-glycans found on TRAP, CSP, and possibly related proteins bearing thrombospondin repeat domains, it is worth considering what role $\mathrm{N}$-glycosylation might play in immune responses to Plasmodium proteins."
\end{abstract}

First draft submitted: 27 November 2017; Accepted for publication: 11 December 2017; Published online: 6 April 2018

Keywords: fucosylation • mannosylation • malaria $\bullet$ RTS,S

Malaria remains a dire public health problem, despite significant efforts to control and eliminate the disease, and is caused by infection with any one of six species of Plasmodium parasite. Although focused control measures have halved the number of annual deaths from malaria to approximately 630,000 [1], the disease is still endemic in 91 countries and resistance to antimalarials continues to spread. Malaria eradication will require a combination of control measures including a vaccine that affords effective protection against different species and strains of Plasmodium parasites; a need that is currently unmet. Although much has been written about the design and evaluation of malaria vaccines, relatively little has been said about the impact that glycosylation has on the efficacy of existing or emerging vaccine technologies. This is probably because the exact nature of protein glycosylation in Plasmodium spp. has been contentious [2,3] and because glycopeptides were for a long time incorrectly thought to be T-independent antigens [4].

Uncertainty surrounding protein glycosylation in Plasmodium parasites was driven in the past by an inability of common glycan-recognizing lectins to bind to parasite proteins and poor histological staining of parasites by periodic acid-Schiff methods. Indeed, it appeared that the installation of glycosylphosphatidylinositol anchors was the only type of glycosylation that Plasmodium parasites undertook [5]. Genome sequencing of Plasmodium spp. also revealed the absence of genes required to build and remodel common eukaryotic glycans [2,3]. Even so, homologs of several genes required for protein glycosylation are present and conserved in the genomes of Plasmodium spp. and metabolomic analyses of parasite material has demonstrated the presence of the nucleotide sugars required for protein glycosylation, which alluded to the existence of as yet undiscovered parasite glycans.

With the aid of modern protein mass spectrometry methods, several research groups have recently tackled the issues of glycan lability and relatively small sample sizes to begin characterizing the true diversity of Plasmodium protein glycosylation [6-12]. Evidence for the synthesis of short N-linked glycans has been reported in asexual blood stages of Plasmodium falciparum [6], and there is every reason to expect that these modifications will be found in other life cycle stages as well. Cell surface proteomic studies of $P$. falciparum and $P$. vivax sporozoites, the two species responsible for the majority of malaria cases and deaths, have demonstrated that $\mathrm{O}$ - and C-linked glycans exist on the essential adhesive proteins, TRAP and CSP $[8,10]$. These abundant surface proteins are required for parasite infectivity and are prime vaccine candidates because sporozoites represent a population bottleneck in the life cycle as parasites pass from mosquito to human and establish an infection in the liver. Moreover, strain-transcending sterile immunity can be achieved through strong B- and T-cell-dependent responses against $P$. falciparum and $P$. vivax liver stages [13-17].

CSP is the target of the furthest-progressed malaria vaccine candidate, GlaxoSmithKline's RTS,S/AS01 $\left(\right.$ Mosquirix $\left.^{\top M}\right)$. In 2015, following a Phase III clinical trial in Africa, this vaccine was approved by the Euro- 
pean Medicines Agency for use in African babies and infants. The vaccine incorporates a recombinant protein comprising several repeat sequences of CSP plus its C-terminal domain (the site that is glycosylated by the parasite) fused to the hepatitis B surface antigen and combined with the proprietary AS01 liposomal adjuvant [18]. RTS,S/AS01 is most effective with four doses and induces both B- and T-cell-mediated immunity. It is the only vaccine that has shown protection against clinical malaria in a Phase III clinical trial, with $18-31 \%$ protection provided to African children [19]. Unfortunately, this protection wanes over time [20], and is frequently followed by rebound cases of malaria [21]. Vaccines based on TRAP, another abundant sporozoite surface protein, have fared no better, with a first-generation T-cell-inducing vaccine proving to be ineffective at reducing the natural infection rate in semi-immune African adults [22]. A variety of factors probably contribute to the suboptimal efficacy of current CSP- and TRAP-based vaccines, but the lack of native glycosylation patterns on the recombinant protein vaccines is likely to be one of these factors.

RTS,S/AS01 works by inducing antibody responses that block sporozoite infection of the liver. The presence or absence of small glycans on Plasmodium surface proteins like CSP has the potential to influence the humoral response to a vaccine like RTS,S/AS01 in several ways. For example, glycosylation can enhance endocytosis of glycoproteins by antigen-presenting cells and protect against excessive proteolysis of glycopeptides within the endolysosome by steric occlusion. If the glycan is small enough, just one or two sugars as is the case for CSP and TRAP, these glycopeptides can be displayed by the MHC II for recognition by the T-cell receptor. Contrary to classical dogma, Kasper and coworkers have shown that glycopeptides are indeed true T-cell epitopes that can drive $\mathrm{CD}^{+}$T-cell help to $\mathrm{B}$ cells for the production of antibodies to the glycosylated antigen [4]. Furthermore, antibodies raised against a glycosylated vaccine should be better able to recognize native sporozoites, particularly if the glycosylated antigen represents an important T-cell epitope. RTS,S/AS01 also induces CD8 ${ }^{+}$T-cell responses in some individuals. Since CSP is presented by MHC I on the surface of infected hepatocytes, and glycopeptides can indeed be presented by MHC I [4], it is possible that glycosylation could also contribute to $\mathrm{CD} 8^{+}$-dependent killing of liver stage parasites.

Beyond the small O- and C-glycans found on TRAP, CSP, and possibly related proteins bearing thrombospondin repeat domains, it is worth considering what role $\mathrm{N}$-glycosylation might play in immune responses to Plasmodium proteins. In P. falciparum, $\mathrm{N}$-glycosylation involves the installation of one or two $\mathrm{N}$-acetyl glucosamine units to asparagine [6] and, as such, may also comprise key T-cell epitopes. Little information is currently available as to which Plasmodium proteins are $\mathrm{N}$-glycosylated, or at what position. Addressing this knowledge gap should be a priority for future proteomic investigations as it will become an important consideration in the design of recombinant protein-based vaccines, including for those targeting blood-stage antigens. Another consideration in vaccine design must surely be whether the glycosylation being incorporated into a vaccine antigen is important or dispensable to the parasite, since the parasite could escape vaccination pressure by modifying or losing the glycosylation, as has been observed in bacteria.

In this regard, the importance of O-glycosylation in the P. falciparum lifecycle was recently investigated [12]. Although all of the genes involved in O-glycosylation, including those encoding the biosynthetic enzymes, the sugar nucleotide transporters, the glycosyltransferases and the substrates, are present and syntenically conserved in Plasmodium spp., the role of O-glycosylation in the parasite lifecycle was unknown. Subsequently, it was shown that O-fucosylation was dispensable in the asexual and sexual blood stages of the $P$. falciparum life cycle, but important for transmission to Anopheles stephensi mosquitoes and for stabilizing thrombospondin repeat-containing proteins for optimal sporozoite motility and liver infectivity in humanized mice [12]. Given the significance of O-glycosylation to $P$. falciparum parasites, the task remains to investigate whether $\mathrm{N}$ - and C-glycosylation is also important, and when, as well as to identify the full repertoire of glycosylated surface proteins across the parasite lifecycle, as these may be new vaccine candidates. Such information will help address which native Plasmodium glycans should be incorporated into recombinant protein-based vaccines in the future.

Building on this, it is worth mentioning that vaccines involving whole parasites, for example, radiation-attenuated sporozoites [13-15] or sporozoites delivered under chemoprotective cover [16,17], will necessarily possess all of the endogenous post-translational modifications of Plasmodium parasites, including glycosylation. Indeed, it is possible that this may contribute, at least in part, to the degree of efficacy reported for these vaccination approaches, and studies into this would be very worthwhile.

Determining how glycosylation impacts different aspects of vaccine efficacy, and to what extent, will require several different approaches. A good starting point would be to determine if patient antibody titres are greater for CSP or TRAP antigens in their glycosylated versus nonglycosylated states, and whether such antibodies are more 
potent at neutralizing sporozoite infectivity. Beyond these experiments, malaria vaccination models will be needed to compare the efficacy of glycosylated and unglycosylated vaccines head-to-head. The conservation of N-, O- and C-glycosylation pathways across Plasmodium spp. suggests that different species and strains can be examined.

The key message we wish to convey is that native post-translational modifications are very important to consider when designing vaccines because they can be incorporated into T-cell epitopes, contrary to classical dogma, in addition to enhancing humoral responses. Modern analytical techniques are identifying more unexpected post-translational 'surprises' and molecular biology is evolving to allow for more rapid study of the roles these modifications play in the parasite's lifecycle. This sets the scene for future elucidation of the importance of glycosylation in basic biology but also for tuning in the efficacy of vaccines against malaria, as well as other important human pathogens.

\section{Financial \& competing interests disclosure}

The authors have no relevant affiliations or financial involvement with any organization or entity with a financial interest in or financial conflict with the subject matter or materials discussed in the manuscript. This includes employment, consultancies, honoraria, stock ownership or options, expert testimony, grants or patents received or pending, or royalties.

No writing assistance was utilized in the production of this manuscript.

\section{References}

1. Gething PW, Casey DC, Weiss DJ et al. Mapping Plasmodium falciparum mortality in Africa between 1990 and 2015. N. Engl. J. Med. 375, 2435-2445 (2016).

2. Von Itzstein M, Plebanski M, Cooke BM, Coppel RL. Hot, sweet and sticky: the glycobiology of Plasmodium falciparum. Trends Parasitol. 24(5), 210-218 (2008).

3. Macedo CS, Schwarz RT, Todeschini AR, Previato JO, Mendonca-Previato L. Overlooked post-translational modifications of proteins in Plasmodium falciparum: N- and O-glycosylation - a review. Mem. Inst. Oswaldo. Cruz 105(8), 949-956 (2010).

4. Avci FY, Li X, Tsuji M, Kasper DL. Carbohydrates and T cells: a sweet twosome. Semin. Immunol. 25(2), 146-151 (2013).

5. Naik RS, Branch OH, Woods AS et al. Glycosylphosphatidylinositol anchors of Plasmodium falciparum: molecular characterization and naturally elicited antibody response that may provide immunity to malaria pathogenesis. J. Exp. Med. 192(11), 1563-1576 (2000).

6. Bushkin GG, Ratner DM, Cui J et al. Suggestive evidence for Darwinian selection against asparagine-linked glycans of Plasmodium falciparum and Toxoplasma gondii. Eukaryot. Cell 9(2), 228-241 (2010).

7. Sanz S, Bandini G, Ospina D et al. Biosynthesis of GDP-fucose and other sugar nucleotides in the blood stages of Plasmodium falciparum. J. Biol. Chem. 288(23), 16506-16517 (2013).

8. Swearingen KE, Lindner SE, Shi L et al. Interrogating the Plasmodium sporozoite surface: identification of surface-exposed proteins and demonstration of glycosylation on CSP and TRAP by mass spectrometry-based proteomics. PLoS Pathog. 12(4), e1005606 (2016).

9. Sanz S, Lopez-Gutierrez B, Bandini G et al. The disruption of GDP-fucose de novo biosynthesis suggests the presence of a novel fucose-containing glycoconjugate in Plasmodium asexual blood stages. Sci. Rep. 6, 37230 (2016).

10. Swearingen KE, Lindner SE, Flannery EL et al. Proteogenomic analysis of the total and surface-exposed proteomes of Plasmodium vivax salivary gland sporozoites. PLoS Negl. Trop. Dis. 11(7), e0005791 (2017).

11. Lopez-Gutierrez B, Dinglasan RR, Izquierdo L. Sugar nucleotide quantification by liquid chromatography tandem mass spectrometry reveals a distinct profile in Plasmodium falciparum sexual stage parasites. Biochem. J. 474(6), 897-905 (2017).

12. Lopaticki S, Yang ASP, John A et al. Protein O-fucosylation in Plasmodium falciparum ensures efficient infection of mosquito and vertebrate hosts. Nat. Comm. 8(1), 561 (2017).

13. Clyde DF, Mccarthy VC, Miller RM, Woodward WE. Immunization of man against falciparum and vivax malaria by use of attenuated sporozoites. Am. J. Trop. Med. Hyg. 24(3), 397-401 (1975).

14. Hoffman SL, Goh LM, Luke TC et al. Protection of humans against malaria by immunization with radiation-attenuated Plasmodium falciparum sporozoites. J. Infect. Dis. 185(8), 1155-1164 (2002).

15. Seder RA, Chang LJ, Enama ME et al. Protection against malaria by intravenous immunization with a nonreplicating sporozoite vaccine. Science 341(6152), 1359-1365 (2013).

16. Roestenberg M, Mccall M, Hopman J et al. Protection against a malaria challenge by sporozoite inoculation. N. Engl. J. Med. 361(5), 468-477 (2009).

17. Mordmuller B, Surat G, Lagler H et al. Sterile protection against human malaria by chemoattenuated PfSPZ vaccine. Nature 542(7642), 445-449 (2017).

18. Rts SCTP, Agnandji ST, Lell B et al. A Phase III trial of RTS,S/AS01 malaria vaccine in African infants. N. Engl. J. Med. 367(24), 2284-2295 (2012). 
19. Rts SCTP. Efficacy and safety of RTS,S/AS01 malaria vaccine with or without a booster dose in infants and children in Africa: final results of a Phase III, individually randomized, controlled trial. Lancet 386(9988), 31-45 (2015).

20. Olotu A, Fegan G, Wambua J et al. Four-year efficacy of RTS,S/AS01E and its interaction with malaria exposure. N. Engl. J. Med. 368(12), 1111-1120 (2013).

21. Olotu A, Fegan G, Wambua J et al. Seven-year efficacy of RTS,S/AS01 malaria vaccine among young African children. N. Engl. J. Med. 374(26), 2519-2529 (2016).

22. Moorthy VS, Imoukhuede EB, Milligan P et al. A randomized, double-blind, controlled vaccine efficacy trial of DNA/MVA ME-TRAP against malaria infection in Gambian adults. PLoS Med. 1(2), e33 (2004). 